UT-911

October 2000

\title{
Volume Preserving Diffeomorphism and Noncommutative Branes
}

\author{
Y. Matsuof and Y. Shibusa \\ Department of Physics, University of Tokyo \\ Bunkyo-ku, Hongo 7-3-1 \\ Tokyo 113-0033 \\ Japan
}

\begin{abstract}
We give a representation of the volume preserving diffeomorphism of $\mathbf{R}^{p}$ in terms of the noncommutative $(p-2)$-branes whose kinetic term is described by the Hopf term. In the static gauge, the $(p-2)$ brane can be described by the free fields and it suggests that the quantization of the algebra is possible.
\end{abstract}

hep-th/0010040

* e-mail address : matsuo@hep-th.phys.s.u-tokyo.ac.jp

$\dagger$ e-mail address : shibusa@hep-th.phys.s.u-tokyo.ac.jp 


\section{Introduction}

In the context of the brane dynamics in the string theory, the volume preserving diffeomorphism of $p$-dimensions $\left(\mathcal{V}_{p}\right)$, infinitesimally generated by

$$
\delta_{v} x^{a}=v^{a}(x), \quad \partial_{a} v^{a}(x)=0,
$$

is the important symmetry which reflects the non-commutativity of the spacetime. The most famous example is the case $p=2$ when the area preserving diffeomorphism $\left(\mathcal{V}_{2}\right)$ is the dynamical degree of freedom in the matrix models [1] [2]. In the BFSS matrix model [1], such a degree of freedom appears as discretization of the residual gauge symmetry of the supermembrane [3]. In the passage from [3] to [1], the quantized algebra of the symmetry was essential to have the D0-brane interpretation. It also appeared in the context of the physics in large B limit and it becomes one of the most actively studied branches in the string theory [4] [5].

Intuitively the generalization to $p>2$ becomes important when the antisymmetric $p$-form field is very large. Such a field is coupled to the volume of the $(p-1)$-branes. When it is large, the configuration space is restricted to the motion which preserves the volume element. In a related but slightly different context, $\mathcal{V}_{p}$ also appears as the residual symmetry after the light-cone gauge fixing [6].

Normally these symmetries have been studied from the viewpoint the Nambu bracket [7] which generalizes the Poisson bracket. This forces us to extend the idea of the phase space and requires a radical change for the quantization. For the recent mathematical studies, see for example [8]. In such works, however, it is not clear to understand the relation with the conventional quantization procedure.

On the other hand, in the discussion of the noncommutativity of $\mathcal{M}$ theory, the $B$ field is replaced by 3rd order antisymmetric tensor $C$ [9]. It is indicated in [10] [11] that as a natural extension of the usual noncommutative geometry, we meet the extended object "noncommutative string" which appears at the end of open membrane.

In this paper, therefore, we use a different route to define the quantization of $\mathcal{V}_{p}$. Instead of redefining the idea of the symplectic structure itself, we use the extended objects $((p-2)$-branes $)$ to describe the symmetry while we use the conventional phase space and the quantization. Unlike the ordinary $D$ branes whose is described by the Dirac-Born-Infeld action, we use the action which is similar to the Hopf term in the nonlinear sigma model. Actually 
such an object appeared long before in the context of the vortex motion in the fluid dynamics for $p=3$ [12].

This paper is organized as follows. In section 2 we summarize the phase structure of the volume preserving diffeomorphism of $p$-dimensions $\left(\mathcal{V}_{p}\right)$. In section 3 we give a description of the noncommutative $(p-2)$-branes and show that they are reduced to a free field theory in the static gauge. We will demonstrate then that a representation of $\mathcal{V}_{p}$ is given in terms of the noncommutative branes in this gauge. In the appendix A we review the derivation of the action for $(p-2)$-branes as the action for the "vortex" of the fluid dynamics. In the appendix $\mathrm{B}$ we give a proof that $\mathcal{V}_{p}$ thus derived is independent of the particular gauge fixing.

\section{Symplectic structure of Volume Preserving Diffeomorphism}

We introduce the symplectic structure of $\mathcal{V}_{p}$ by the coadjoint orbit method [13]. The Lie algebra of $\mathcal{V}_{p}$ (which we denote $g$ ) is defined by the commutation relation between two vector fields in (1),

$$
[v, w]^{a}=v^{b} \partial_{b} w^{a}-w^{b} \partial_{b} u^{a}
$$

where and in the following $a, b$ runs from 1 to $p$. We use $U_{a}(x)$ which satisfies $\partial_{a} U^{a}=0$ as representing the element in $g^{*}$ with the inner product,

$$
\langle U, v\rangle \equiv U_{v} \equiv \int d^{p} x U_{a}(x) v^{a}(x) .
$$

The symplectic structure on the coadjoint orbit implies that we have the following Poisson bracket between $U$,

$$
\left\{U_{a}(x), U_{b}(y)\right\}=\left(\partial_{b} U_{a}(x)-\partial_{a} U_{b}(x)\right) \delta^{(p)}(x-y),
$$

which can be rewritten in terms of $U_{v}$ as,

$$
\left\{U_{v}, U_{w}\right\}=U_{[v, w]}
$$

The last equation explicitly shows that (4) gives the representation of the algebra (2). 
In the dynamics of the perfect fluid, this Poisson bracket gives the Hamiltonian formalism of the Navier-Stokes equation. Indeed if we choose

$$
H=\frac{1}{2} \int d^{p} x U_{a}(x) U^{a}(x),
$$

as the Hamiltonian. Then the equation of motion for $U$ is given by the Navier-Stokes equation,

$$
\frac{\partial U^{a}}{\partial t}=\left\{H, U^{a}\right\}=U^{b} \partial^{a} U_{b}-U^{b} \partial_{b} U^{a} .
$$

In this sense, the Navier-Stokes equation describes a straight line in $\mathcal{V}_{p}$ and the Poisson bracket (四) describes the symplectic structure of the velocity fields.

As we review in the appendix, the noncommutative brane describes the origin of the codimension two vortex in $\mathbf{R}^{p}$. In this context, it is more convenient to rewrite the Poisson bracket in terms of the vorticity $(p-2)$ forms,

$$
\omega^{a_{1} \cdots a_{p-2}}(x) \equiv \frac{1}{(p-2) !} \epsilon^{a_{1} \cdots a_{p}} \partial_{a_{p-1}} U_{a_{p}} .
$$

We write the generators of $\mathcal{V}_{p}$ in terms of $\omega$ as,

$$
\omega_{f} \equiv \int d^{p} x \epsilon_{a_{1} \cdots a_{p}} \omega^{a_{1} \cdots a_{p-2}}(x) f^{a_{p-1} a_{p}}(x) .
$$

$\omega_{f}$ is related to $U_{v}$ as,

$$
\omega_{f}=U_{v}, \quad \text { with } \quad v^{a}(x)=-2 \partial_{b} f^{b a} .
$$

It is clear that the vector field $v^{a}$ thus defined from $f$ automatically satisfies $\partial_{a} v^{a}=0$. There are, however, some arbitrariness to define $f$ from $v$. To fix it, we require that the two form $f_{a b} d x^{a} d x^{b}$ satisfies the equation $d f=0$. Together with the defining equation $-2 \delta f=v(\delta=* d *$ with $*$ as Hodge dual), one can determine $f$ from $v$ as (up to the harmonic two forms),

$$
f_{a b}(x)=\frac{1}{2} \int d^{p} y G^{(p)}(x, y)\left(\frac{\partial}{\partial y^{a}} v_{b}(y)-\frac{\partial}{\partial y^{b}} v_{a}(y)\right),
$$

with the Green function $\partial_{x}^{2} G^{(p)}(x, y)=-\delta^{(p)}(x-y)$. In terms of $\omega_{f}$, the Poisson bracket (田) is replaced by,

$$
\begin{aligned}
\left\{\omega_{f}, \omega_{g}\right\} & =\omega_{\hat{[} f, g]}, \\
(\hat{[} f, g])^{a b} & \equiv 2 \partial_{c} f^{c b} \partial_{d} g^{d a}-2 \partial_{c} f^{c a} \partial_{d} g^{d b} .
\end{aligned}
$$


It is easy to check the consistency condition between two commutators, $\left.-2 \partial_{b}(\hat{[} f, g]\right)^{b a}=([v, w])^{a}$ if $-2 \partial_{b} f^{b a}=v^{a}$ and $-2 \partial_{b} g^{b a}=w^{a}$. Although (4) directly defines the volume preserving diffeomorphism, we will mainly use (11) since the vorticity has much more direct interpretation in terms of the non-commutative branes.

When $p=2$, (11) has a more familiar form. We rewrite $f^{a b}(x)=\epsilon_{a b} f$ and so on, the commutator (11) reduces to,

$$
(\hat{[f}, g \hat{]})^{a b}=\{f, g\} \epsilon^{a b}, \quad\{f, g\}=\epsilon^{a b} \partial_{a} f \partial_{b} g
$$

which is the conventional Poisson bracket for the coordinates which produces the area-preserving diffeomorphism.

\section{Theory of Noncommutative Branes}

The starting point of our discussion for the noncommutative $(p-2)$-branes embedded in $\mathbf{R}^{p}$ is the action of the following form,

$$
S_{0}=\frac{-1}{p} \sum_{I=1}^{N} \Gamma_{I} \int d \sigma_{I} d t \epsilon_{a_{1} \cdots a_{p}} X_{I}^{a_{1}} \frac{\partial X_{I}^{a_{2}}}{\partial t} \epsilon^{i_{1} \cdots i_{p-2}} \frac{\partial X_{I}^{a_{3}}}{\partial \sigma_{I}^{i_{1}}} \cdots \frac{\partial X_{I}^{a_{p}}}{\partial \sigma_{I}^{i_{p-2}}},
$$

where $\sigma_{I}^{i}$ are the coordinates for the spatial direction of $I$-th $(p-2)$-brane. Here we wrote only the kinetic term which governs the phase space. One may add potential terms which do not contain the time derivative of $X$ to (13) without changing the algebra.

We first recall how such an action appeared in the context of string theory. For $p=2$, it describes the motion of the end points of the open string on the D-brane [14,

$$
S=\frac{1}{2} \int_{\Sigma} d^{2} \sigma B_{a b} \epsilon_{\alpha \beta} \partial_{\alpha} X^{a} \partial_{\beta} X^{b}=\frac{1}{2} \int_{\partial \Sigma} d t B_{a b} X^{a} \partial_{t} X^{b}
$$

If $B$ field is block diagonalized, $\Gamma_{I}$ is identified with $I$-th block of $B=\operatorname{diag}\left(\Gamma_{1} \epsilon, \cdots \Gamma_{N} \epsilon\right)$ with $\epsilon=\left(\begin{array}{cc}0 & 1 \\ -1 & 0\end{array}\right)$. Similarly, for $p=3$, such a kinetic term appeared in the description of "noncommutative string" [10 11 |11 which describes the stringy degree of freedom appearing at the boundary of the open membrane world volume. In this case $\Gamma_{I}$ is related to the magnitude 
of the 3rd order anti-symmetric tensor in $\mathcal{M}$-theory. The $(p-2)$-branes which is described by the action (13) is a generalization of these extended noncommutative objects. In this context we call them as "noncommutative p-brane".

The noncommutative $p$-brane for $p=0,1$ actually appeared long before in the literature of the fluid dynamics. As we mentioned in the previous section, the volume preserving diffeomorphism is the configuration space for the perfect fluid and Euler's equation describes the straight line in $\mathcal{V}_{p}$ [13. The noncommutative brane of codimension two describes the vortex which keeps its form under time evolution, and therefore it is possible to derive the action which describes its own motion. See the appendix A for the derivation of the kinetic term (13). For $p=3$, such an action was discussed in the classic paper [12]. For the geometrical and dynamical implication of the Hopf term, see for example [15] 16.

Let us proceed to the analysis of the symplectic structure defined by $S_{0}$. Since $S_{0}$ contains only one time derivative, the momentum variable is written in terms of $X$,

$$
\Pi_{I a}\left(\sigma_{I}\right) \equiv \frac{\partial S_{0}}{\partial \dot{X}_{I}^{a}}\left(\sigma_{I}\right)=\frac{\Gamma_{I}}{p} \epsilon_{a b a_{1} \cdots a_{p-2}} X_{I}^{b} \epsilon^{i_{1} \cdots i_{p-2}} \frac{\partial X_{I}^{a_{1}}}{\partial \sigma_{I}^{i_{1}}} \cdots \frac{\partial X_{I}^{a_{p-2}}}{\partial \sigma_{I}^{i_{p-2}}}\left(\sigma_{I}\right) .
$$

Since the conjugate variable is written by the original fields, we need to impose the primary constraints,

$$
\phi_{I a}\left(\sigma_{I}\right)=\Pi_{I a}\left(\sigma_{I}\right)-\frac{\Gamma_{I}}{p} \epsilon_{a b a_{1} \cdots a_{p-2}} X_{I}^{b} \epsilon^{i_{1} \cdots i_{p-2}} \frac{\partial X_{I}^{a_{1}}}{\partial \sigma_{I}^{i_{1}}} \cdots \frac{\partial X_{I}^{a_{p-2}}}{\partial \sigma_{I}^{i_{p-2}}}\left(\sigma_{I}\right) \approx 0 .
$$

By using the canonical commutation relation,

$$
\left\{X_{I}^{a}\left(\sigma_{I}\right), \Pi_{J b}\left(\sigma_{J}^{\prime}\right)\right\}=\delta_{I J} \delta_{b}^{a} \delta^{(p-2)}\left(\sigma_{I}-\sigma_{J}^{\prime}\right),
$$

the Poisson bracket between $\phi_{I a}$ is given by,

$$
\begin{aligned}
\left\{\phi_{I a}\left(\sigma_{I}\right), \phi_{J b}\left(\sigma_{J}^{\prime}\right)\right\}= & -\Gamma_{I} \delta_{I J} \epsilon_{a b a_{1} \cdots a_{p-2}} \epsilon^{i_{1} \cdots i_{p-2}} \frac{\partial X_{I}^{a_{1}}}{\partial \sigma_{I}^{i_{1}}} \cdots \frac{\partial X_{I}^{a_{p-2}}}{\partial \sigma_{I}^{i_{p-2}}} \\
& \cdot \delta^{(p-2)}\left(\sigma_{I}-\sigma_{I}^{\prime}\right) \\
\equiv & M_{a b}^{I} \delta_{I J} \delta^{(p-2)}\left(\sigma_{I}-\sigma_{I}^{\prime}\right) .
\end{aligned}
$$

Since $M_{a b}^{I} \frac{\partial X_{I}^{b}}{\partial \sigma_{I}^{2}}=0$ for $i=1,2, \cdots, p-2$, the matrix $M$ has rank 2. We therefore expect to have $p-2$ first class constraints which correspond to the reparametrization of the world volume coordinates $\sigma$. 
Indeed if we combine them into $T_{I i}=-\frac{\partial X_{I}^{a}}{\partial \sigma_{I}^{2}} \phi_{I a}$, they satisfy the algebra,

$$
\begin{aligned}
\left\{T_{I i}\left(\sigma_{I}\right), T_{J j}\left(\sigma_{J}^{\prime}\right)\right\}= & \delta_{I J} T_{I i}\left(\sigma_{I}\right) \frac{\partial}{\partial \sigma_{I}^{j}} \delta^{(p-2)}\left(\sigma_{I}-\sigma_{J}^{\prime}\right) \\
& +\delta_{I J} T_{I j}\left(\sigma_{I}\right) \frac{\partial}{\partial \sigma_{I}^{i}} \delta^{(p-2)}\left(\sigma_{I}-\sigma_{J}^{\prime}\right) \\
& +\delta_{I J} \frac{\partial T_{I i}\left(\sigma_{I}\right)}{\partial \sigma_{I}^{j}} \delta^{(p-2)}\left(\sigma_{I}-\sigma_{J}^{\prime}\right),
\end{aligned}
$$

which is the generalization of the classical Virasoro algebra to the higher dimensions. The existence of the first class constraints forces us to introduce some extra constraints to fix the gauge.

There are several possible choices for the gauge fixing. The choice we took in this paper is the static gaug日 defined by,

$$
\chi_{I}^{i} \equiv X_{I}^{i+2}\left(\sigma_{I}\right)-\sigma_{I}^{i} \approx 0,
$$

where (and in the following) $i$ takes its value in the range $1, \cdots, p-2$. It was shown that the set of constraints $\left\{\xi_{\alpha}\right\}=\left\{\phi_{I a}\left(\sigma_{I}\right), \chi_{J}^{i}\left(\sigma_{J}\right)\right\},(\alpha$ represents the indices $\left.I, i, a, \sigma_{I}\right)$ becomes the second class and we can define the Dirac bracket as,

$$
[F, G]_{D}=\{F, G\}-\sum_{\alpha, \beta}\left\{F, \xi_{\alpha}\right\}\left(C^{-1}\right)^{\alpha \beta}\left\{\xi_{\beta}, G\right\}
$$

where the matrix $C$ is defined as $C_{\alpha \beta}=\left\{\xi_{\alpha}, \xi_{\beta}\right\}$. In this gauge choice, we obtain

$$
\begin{aligned}
C^{-1}\left[\chi_{I i}\left(\sigma_{I}\right), \phi_{J}^{a}\left(\tilde{\sigma}_{J}\right)\right] & =-\delta_{I J} \frac{\partial X_{I}^{a}\left(\tilde{\sigma}_{I}\right)}{\partial \tilde{\sigma}_{I}^{i}} \Theta\left(\tilde{\sigma}_{I}^{i}-\sigma_{I}^{i}\right) \prod_{j=1}^{i-1} \prod_{j=i+1}^{p-2} \delta\left(\sigma_{I}^{j}-\tilde{\sigma}_{I}^{j}\right), \\
C^{-1}\left[\phi_{I}^{a}\left(\sigma_{I}\right), \phi_{J}^{b}\left(\tilde{\sigma}_{J}\right)\right] & =\frac{1}{\Gamma_{I}(p-2) !} \epsilon^{a b 34 \cdots p} \delta_{I J} \delta^{(p-2)}\left(\sigma_{I}-\tilde{\sigma}_{J}\right) \\
C^{-1}\left[\chi_{I i}\left(\sigma_{I}\right), \chi_{J j}\left(\tilde{\sigma}_{J}\right)\right] & =0 .
\end{aligned}
$$

It gives the bracket of the free field theory,

$$
\begin{aligned}
{\left[X_{I}^{1}\left(\sigma_{I}\right), X_{J}^{2}\left(\sigma_{J}^{\prime}\right)\right]_{D} } & =\frac{1}{\Gamma_{I}(p-2) !} \delta^{(p-2)}\left(\sigma_{I}-\sigma_{J}^{\prime}\right) \delta_{I J}, \\
{\left[X_{I}^{i+2}\left(\sigma_{I}\right), X_{J}^{a}\left(\sigma_{J}^{\prime}\right)\right]_{D} } & =0 .
\end{aligned}
$$

\footnotetext{
${ }^{1}$ For $O(3)$ invariant but nonlinear constraints for $p=3$, see 10$][1]$ [18].
} 
Actually the reduction to the free theory can be easily seen at the Lagrangian level by putting the constraint (20) to the action (13). By just inspection, one can understand that it reduces to the quadratic in terms of $X$ and thus define a free theory. It reminds us of the fact that the ordinary string theory was defined by the nonlinear Nambu-Goto action but it reduces to the free theory in the light-cone gauge.

As we explain the appendix, the vorticity is related to the embedding functions of the $(p-2)$-brane as,

$$
\omega^{a_{1} \cdots a_{p-2}}(x) \equiv \sum_{I=1}^{N} \Gamma_{I} \int d^{p-2} \sigma_{I} \epsilon^{i_{1} \cdots i_{p-2}} \frac{\partial X_{I}^{a_{1}}}{\partial \sigma_{I}^{i_{1}}} \cdots \frac{\partial X_{I}^{a_{p-2}}}{\partial \sigma_{I}^{i_{p-2}}} \delta^{(p)}\left(x-X_{I}\left(\sigma_{I}\right)\right) .
$$

We identify the generator of the volume preserving diffeomorphism of $p$ dimensions $\left(\mathcal{V}_{p}\right)$ as follows,

$$
\begin{aligned}
\omega_{f} & \equiv \int d^{p} x \epsilon_{a_{1} \cdots a_{p}} \omega^{a_{1} \cdots a_{p-2}}(x) f^{a_{p-1} a_{p}}(x), \\
& =\sum_{I=1}^{N} \Gamma_{I} \int d \sigma_{I} \epsilon_{a_{1} \cdots a_{p-2}} \epsilon^{i_{1} \cdots i_{p-2}} \frac{\partial X_{I}^{a_{1}}}{\partial \sigma_{I}^{i_{1}}} \cdots \frac{\partial X_{I}^{a_{p-2}}}{\partial \sigma_{I}^{i_{p-2}}} f^{a_{p-1} a_{p}}\left(X_{I}\left(\sigma_{I}\right)\right) .
\end{aligned}
$$

Indeed it generates $\mathcal{V}_{p}$ on $X$,

$$
\begin{aligned}
{\left[X_{I}^{a}\left(\sigma_{I}\right), \omega_{f}\right]_{D} } & =-2 \partial_{b} f^{b a}\left(X_{I}\left(\sigma_{I}\right)\right)+\sum_{i=1}^{p-2} 2 \partial_{b} f^{b i+2}\left(X_{I}\left(\sigma_{I}\right)\right) \frac{\partial X_{I}^{a}}{\partial \sigma_{I}^{i}} \\
& =v^{a}\left(X_{I}\left(\sigma_{I}\right)\right)+\left.\frac{d}{d t} X^{a}\left(\sigma_{I}^{i}-t v^{i+2}\left(X_{I}\left(\sigma_{I}\right)\right)\right)\right|_{t=0} .
\end{aligned}
$$

The second term in the right hand side of $(26)$ comes from the reparametrization because the gauge choice (20) must be preserved. In the second line, we show that it can be absorbed in the infinitesimal reparametrization of the world volume coordinates.

From (23), one can show by the direct calculation that $\omega_{f}$ satisfies the algebra of $\mathcal{V}_{p}$,

$$
\left[\omega_{f}, \omega_{g}\right]_{D}=-\omega_{\hat{[f, g]}} .
$$

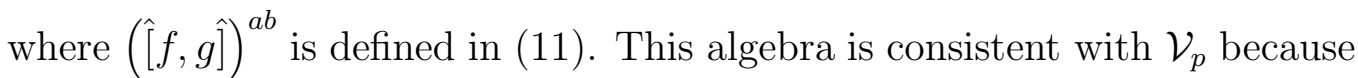
in this system

$$
\begin{aligned}
\left(\delta_{f} \delta_{g}-\delta_{g} \delta_{f}\right) X^{a} & =\left[\left[X^{a}, \omega_{g}\right]_{D}, \omega_{f}\right]_{D}-\left[\left[X^{a}, \omega_{f}\right]_{D}, \omega_{g}\right]_{D} \\
& =\left[X^{a},\left[\omega_{g}, \omega_{f}\right]_{D}\right]_{D} .
\end{aligned}
$$


Since $\omega_{f}$ is invariant under the reparametrization of $\sigma$, we expect that this result does not depend on the gauge choice. We give some further reasoning for the gauge independence in appendix B.

At this point, it seems attractive if one may have a mapping from arbitrary functions on $\mathbf{R}^{p}$ to the functional of the brane coordinates such that we have the following relation,

$$
F(x) \in C\left(\mathbf{R}^{p}\right) \rightarrow \mathcal{O}_{F}, \quad \text { such that } \quad\left\{\mathcal{O}_{F}, \omega_{f}\right\}_{D}=\mathcal{O}_{\delta_{f} F}
$$

A plausible candidate is, $\mathcal{O}_{F}=\int d^{p-2} \sigma F(X(\sigma))$. This is not, unfortunately, so straightforward. As we explicitly wrote in (27), there are extra term in the Dirac bracket which can be only preserved in the reparametrization of $\sigma$. The expression for $\mathcal{O}_{F}$ can transform covariantly only when $F(X(\sigma))$ transforms as $d^{p-2} \sigma F(X(\sigma))=d^{p-2} \tilde{\sigma} F(X(\tilde{\sigma}))$. Such a transformation is possible only if $F$ is $(p-2)$ - (or Hodge dual 2-) form in the target space. In this case, they are actually the generators of $\mathcal{V}_{p}$ themselves. To define the covariant functional of the general $q$-forms, we need to introduce the world volume metric tensor.

\section{Discussions}

In this paper, we gave the classical symplectic structure of the volume preserving diffeomorphism while not explicitly attempted to quantize the symmetry. The symplectic structure for the noncommutative branes turned out to be very simple in the static gauge and can be quantized immediately. For

$p=3$ case as an example, the quantized version of the Dirac bracket (23) gives the commutation relation

$$
\left[X^{1}(\sigma), X^{2}\left(\sigma^{\prime}\right)\right]=\delta\left(\sigma-\sigma^{\prime}\right)
$$

This commutation relation is the same as the bosonic ghost in the superstring theory. The generator of the volume preserving diffeomorphism takes the following form,

$$
\omega_{f}=\int d \sigma\left(f^{12}(X)+f^{23}(X) \frac{\partial X^{1}}{\partial \sigma}+f^{31}(X) \frac{\partial X^{2}}{\partial \sigma}\right) .
$$

Somewhat similar construction of space-time reparametrization symmetry appeared in [19] where generators are written in terms of $\beta-\gamma$ system. 
Unlike that situation, the generators of $\mathcal{V}_{3}$ are nonlinear both in $\beta$ and $\gamma$ and highly singular. One obvious difficulty is how to organize the regularization scheme such that we can keep the desirable symmetry such as $O(3)$. The other, but maybe related, issue for $p=3$ case is the BRST invariance. These considerations may give the constraints on the target space.

Another issue is how to introduce the target space supersymmetry. In 10 [11], these noncommutative strings are introduced to describe the noncritical self-dual string in six dimensions [20]. Supersymmetry will be indispensable to give some insights to these issues from our viewpoint.

Acknowledgements: One of the author (YM) would like to thank K. Fujikawa, S. Yahikozawa, and D. Minic for the comments and discussions. He would also like to thank I. Bars for his hospitality during the stay at the CIT-USC center where the final part of this draft was typed.

YM is supported in part by Grant-in-Aid (\# 09640352) and in part by Grantin-Aid for Scientific Research in a Priority Area, "Supersymmetry and Unified Theory of Elementary Particle" (\# 707) from the Ministry of Education, Science, Sports and Culture.

\section{A Vortices in Ideal Fluid}

In fluid dynamics without viscosity, it is known that the vortex configuration of codimension 2 (at least for $p=2,3$ ),

$$
\omega^{a_{1} \cdots a_{p-2}}(x)=\sum_{I=1}^{N} \Gamma_{I} \int d \sigma_{I} \epsilon^{i_{1} \cdots i_{p-2}} \frac{\partial X_{I}^{a_{1}}}{\partial \sigma_{I}^{i_{1}}} \cdots \frac{\partial X_{I}^{a_{p-2}}}{\partial \sigma_{I}^{i_{p-2}}} \delta^{(p)}\left(x-X_{I}\left(\sigma_{I}\right)\right) .
$$

keeps its form under the time evolution. To show it, we derive the vector field which produces the vorticity as,

$$
\begin{aligned}
U_{a}(x)= & (-1)^{p} \sum_{I=1}^{N} \Gamma_{I} \int d \sigma_{I} \epsilon_{a a_{1} \cdots a_{p-2} b} \\
& \cdot \epsilon^{i_{1} \cdots i_{p-2}} \frac{\partial X_{I}^{a_{1}}}{\partial \sigma_{I}^{i_{1}}} \cdots \frac{\partial X_{I}^{a_{p-2}}}{\partial \sigma_{I}^{i_{p-2}}} \partial^{b} G^{(p)}\left(x-X_{I}\left(\sigma_{I}\right)\right),
\end{aligned}
$$

with the Green function defined after (10). If we put these expression into the Navier-Stokes equation (6), one may show that a consistent solution can 
be found by keeping the vortex configuration itself while the location of the vortex is changed by the equation

$$
\epsilon_{a_{1} \cdots a_{p}} \frac{\partial X_{I}^{a_{1}}}{\partial \sigma_{I}^{1}} \cdots \frac{\partial X_{I}^{a_{p-2}}}{\partial \sigma_{I}^{p-2}}\left(U^{a_{p-1}}\left(X_{I}\right)-\frac{\partial X_{I}^{a_{p-1}}}{\partial t}\right)=0,
$$

or by solving it,

$$
\frac{\partial X_{I}^{a}\left(t, \sigma_{I}\right)}{\partial t}=U^{a}\left(X_{I}\left(\sigma_{I}\right)\right)+\sum_{i=1}^{p-2} \alpha_{I}^{i} \frac{\partial X_{I}^{a}\left(t, \sigma_{I}\right)}{\partial \sigma_{I}^{i}} .
$$

Here $\alpha_{I}^{i}$ is the arbitrary parameter. The second term represents the time evolution along the brane and can be absorbed into the time dependent reparametrization of world brane coordinates $\sigma_{I}^{i}$.

Originally the Navier-Stokes equation has the phase space described by $U^{a}$, the above statement shows that it can be consistently truncated to the much smaller degree of freedom, namely the locations of $(p-2)$-branes. It is then natural to suspect that there is the action for $X$ which directly gives (35). Such an equation has been known for $p=2,3$ [12], and we give the higher dimensional extension here. Indeed it is the combination of the kinetic term (13) and the potential term which is specific of the Navier-Stokes equation,

$$
\begin{aligned}
S= & S_{0}-H \\
H= & \frac{1}{2} \sum_{I, J=1}^{N} \Gamma_{I} \Gamma_{J}(p-2) ! \int d \sigma_{I} d \sigma_{J} \epsilon^{i_{1} \cdots i_{p-2}} \frac{\partial X_{I}^{a_{1}}}{\partial \sigma_{I}^{i_{1}}} \cdots \frac{\partial X_{I}^{a_{p-2}}}{\partial \sigma_{I}^{i_{p-2}}} \\
& \epsilon^{j_{1} \cdots j_{p-2}} \frac{\partial X_{J a_{1}}}{\partial \sigma_{J}^{j_{1}}} \cdots \frac{\partial X_{J a_{p-2}}}{\partial \sigma_{J}^{i_{p-2}}} G^{(p)}\left(X_{I}\left(\sigma_{I}\right)-X_{J}\left(\sigma_{J}\right)\right) \\
S_{0}= & -\frac{1}{p} \sum_{I=1}^{N} \Gamma_{I} \int d \sigma_{I} d t \epsilon_{a_{1} \cdots a_{p}} X_{I}^{a_{1}} \frac{\partial X_{I}^{a_{2}}}{\partial t} \epsilon^{i_{1} \cdots i_{p-2}} \frac{\partial X_{I}^{a_{3}}}{\partial \sigma_{I}^{i_{1}}} \cdots \frac{\partial X_{I}^{a_{p}}}{\partial \sigma_{I}^{i_{p-2}}} .
\end{aligned}
$$

The variation of this action gives (35).

Appearance of the undetermined parameters in (36) can be explained by solving the constrained system. The Hamiltonian for $(p-2)$-branes, $H$, should be modified by including the constraints (total Hamiltonian),

$$
H_{T} \equiv H+\sum_{I=1}^{N} \int d \sigma_{I} \lambda_{I}^{a}\left(\sigma_{I}\right) \phi_{I a}\left(\sigma_{I}\right)
$$


From the consistency condition $\dot{\phi_{I a}}=\left\{\phi_{I a}, H_{T}\right\} \approx 0$, we obtain

$$
\lambda_{I}^{a}\left(\sigma_{I}\right)=u^{a}\left(X_{I}\left(\sigma_{I}\right)\right)+\sum_{i=1}^{p-2} \alpha^{i}\left(\sigma_{I}\right) \frac{\partial X_{I}^{a}\left(\sigma_{I}\right)}{\partial \sigma_{I}^{i}} .
$$

Here $\alpha^{i}\left(\sigma_{I}\right)$ are undetermined functions. This ambiguity comes from the reparametrization invariance. It is fixed by the gauge conditions $(20)$,

$$
\alpha^{i}\left(\sigma_{I}\right)=-u^{i+2}\left(X_{I}\left(\sigma_{I}\right)\right)+a^{i}
$$

where $a^{i}$ are constant parameters which correspond to $p$-brane translation.

\section{B Gauge independence of $\mathcal{V}_{p}$}

In this appendix, we give a confirmation that the algebra $(28)$ does not depend on the gauge fixing condition (20). It may be obvious that the Dirac bracket between the gauge invariant quantities does not depend on the particular gauge choice. However, since we do not know the explicit proof of this statement, we give a (admittingly insufficient) calculation which support it.

We change the gauge fixing condition infinitesimally by,

$$
\begin{aligned}
& \chi_{I}^{i} \equiv X_{I}^{i+2}\left(\sigma_{I}\right)-\sigma_{I}^{i} \approx 0, \quad(i=2, \cdots, p-2) \\
& \chi_{I}^{3} \equiv X_{I}^{3}\left(\sigma_{I}\right)-\sigma_{I}^{1}-\epsilon h\left(X_{I}\left(\sigma_{I}\right), \sigma_{I}\right) \approx 0 .
\end{aligned}
$$

Up to the first order of $\epsilon$, the Dirac bracket between $X$ is modified to

$$
\begin{aligned}
{\left[X_{I}^{1}\left(\sigma_{I}\right), X_{J}^{2}\left(\sigma_{J}^{\prime}\right)\right]_{D}=} & \frac{1}{\Gamma_{I}(p-2) !} \delta^{(p-2)}\left(\sigma_{I}-\sigma_{J}^{\prime}\right) \delta_{I J} \\
& +\epsilon \frac{1}{\Gamma_{I}(p-2) !} \delta^{(p-2)}\left(\sigma_{I}-\sigma_{J}^{\prime}\right) \delta_{I J}\left(-\frac{\partial h}{\partial \sigma_{I}^{1}}-\frac{\partial h}{\partial X_{I}^{3}}\right), \\
{\left[X_{I}^{1}\left(\sigma_{I}\right), X_{J}^{3}\left(\sigma_{J}^{\prime}\right)\right]_{D}=} & \epsilon \frac{1}{\Gamma_{I}(p-2) !} \delta^{(p-2)}\left(\sigma_{I}-\sigma_{J}^{\prime}\right) \delta_{I J}\left(\frac{\partial h}{\partial X_{I}^{2}}\right) \\
{\left[X_{I}^{2}\left(\sigma_{I}\right), X_{J}^{3}\left(\sigma_{J}^{\prime}\right)\right]_{D}=} & \epsilon \frac{1}{\Gamma_{I}(p-2) !} \delta^{(p-2)}\left(\sigma_{I}-\sigma_{J}^{\prime}\right) \delta_{I J}\left(-\frac{\partial h}{\partial X_{I}^{1}}\right) \\
\text { Others }= & 0 .
\end{aligned}
$$

We confirmed that the algebra (28) does not change to the first order in $\epsilon$ with this modified brackets. 


\section{References}

[1] T. Banks, W. Fischler, S. H. Shenker, L. Susskind, Phys. Rev. D55 (1997) 5112-5128 [hep-th/9610043].

[2] N. Ishibashi, H. Kawai, Y. Kitazawa, A. Tsuchiya, Nucl. Phys. B498 (1997) 467-491 [hep-th/9612115].

[3] B. de Wit, J. Hoppe, H. Nicolai, Nucl. Phys. B305 (1988) 545.

[4] A. Connes, M. R. Douglas, A. Schwarz, JHEP 9802 (1998) 003 [hep-th/9711162].

[5] N. Seiberg and E. Witten, JHEP 9909 (1999) 032 [hep-th/9908142].

[6] E. Bergsheff, E. Sezgin, Y. Tanii and P. K. Townsend, Annals of Physics 199 (1990) 340.

[7] Y. Nambu, Phys. Rev. D7 (1973) 2405.

[8] L. Takhtajan, Comm. Math. Phys. 160 (1994) 295;

G. Dito, M. Flato, D. Sternheimer, L. Takhtajan, Commun. Math. Phys. 183 (1997) 1.

[9] C-S. Chu, P-H. Ho and M. Li, Nucl. Phys. B574 (2000) 275-287 [hep-th/9911153].

[10] E. Bergshoeff, D.S. Berman, J.P. van der Schaar, P. Sundell, " Noncommutative M Theory Five-brane" [hep-th/0005026].

[11] S. Kawamoto and N. Sasakura, JHEP 0007 (2000) 014 [hep-th/0005123].

[12] F. Lund and T. Regge, Phys. Rev. D14 (1976) 1524.

[13] See for example, V. Arnold, "Mathematical Methods in Classical Mechanics", Springer Verlag (1989), Appendix 2.

[14] C-S. Chu, P-M. Ho, Nucl. Phys. (1999) B550 151-168 [hep-th/9812219].

[15] A. M. Polyakov, Mod. Phys. Lett. A3 (1988) 325. 
[16] Y.S. Wu, A. Zee, Phys. Lett. B207 (1988) 39;

C.-H. Tze, Int. J. Mod. Phys. A3 (1988) 1959;

C.-H. Tze, S. Nam, Annals Phys. 193 (1989) 419.

[17] H. Awata, M. Li, D. Minic and T. Yoneya, "On the quantization of Nambu brackets" [hep-th/9906248].

[18] Y. Matsuo, Mod. Phys. Lett. A8 (1993) 2677 [hep-th/9305151].

[19] A. Giveon, D. Kutasov and N. Seiberg, Adv. Theor. Math. Phys. 2 (1998) 733, hep-th/9806194] .

[20] E. Witten, "Some comments on the string dynamics" [hep-th/9507121];

N. Seiberg and E. Witten, Nucl. Phys. B471 (1996) 121 [hep-th/9603003]. 